\title{
Zinc montmorillonite as a reusable heterogeneous catalyst for the synthesis of 2,3-dihydro-1H-1,5-benzodiazepine derivatives
}

\author{
Ravi Varala, Ramu Enugala, and Srinivas R. Adapa* \\ Indian Institute of Chemical Technology, Hyderabad, 500 007, India \\ E-mail:rvarala_iict@yahoo.co.in
}

\begin{abstract}
A novel approach for the synthesis of 2,3-dihydro-1H-1,5-benzodiazepines from $o$-phenylenediamines and structurally divergent ketones is described using zinc montmorillonite as catalyst at room temperature. The catalyst can be reused for at least three cycles with consistent activity.
\end{abstract}

Keywords: 2,3-Dihydro-1H-1,5-benzodiazepines, zinc montmorillonite, ketones, $o$-phenylenediamines

CAUTION: $o$-Phenylenediamines are toxic to work with and hazardous to the environment

\section{Introduction}

1,5-Benzodiazepine derivatives have received significant attention, and the core is indeed a "privileged scaffold" found in compounds active against a variety of target types including peptide hormones (such as CCK), ${ }^{1 \mathrm{a}}$ interleukin converting enzymes (ICE) ${ }^{1 \mathrm{~b}}$ and potassium blockers $\left(\mathrm{I}_{\mathrm{k}}\right)^{1 \mathrm{c}}$. More recently, the area of biological interest of 1,5-benzodiazepines has been extended to various diseases such as cancer, ${ }^{2 a}$ viral infection (non-nucleoside inhibitors of HIV-1 reverse transcriptase), ${ }^{2 \mathrm{~b}, 2 \mathrm{e}}$ cardiovascular disorders. $^{2 \mathrm{c}-\mathrm{d}}$ In addition, 1,5-benzodiazepines show antidepressive, antifungal, antibacterial, antifeedant, antiinflammatory, analgesic and anticonvulsant activities. ${ }^{3}$ Besides, these derivatives are also used as dyes for acrylic fibre ${ }^{4}$ in photography. Moreover, 1,5-benzodiazepines are valuable synthons used for the preparation of other fused ring compounds such as triazolo-, oxadiazolo-, oxazino-, or furanobenzodiazepines. ${ }^{5}$ Despite their importance from a pharmacological, industrial and synthetic point of view, comparatively few methods for their preparation are reported in the literature, a great number of which have appeared only very recently employing $\mathrm{BF}_{3} \cdot \mathrm{Et}_{2} \mathrm{O}$, ${ }^{6 \mathrm{a}} \mathrm{NaBH}_{4},{ }^{6 \mathrm{~b}}$ polyphosphoric acid or $\mathrm{SiO}_{2}{ }^{6 \mathrm{c}} \mathrm{MgO} / \mathrm{POCl}_{3},{ }^{6 \mathrm{~d}} \mathrm{Yb}(\mathrm{OTf})_{3},{ }^{6 \mathrm{e}} \mathrm{Al}_{2} \mathrm{O}_{3} / \mathrm{P}_{2} \mathrm{O}_{5}$ or $\mathrm{AcOH}$ under microwave conditions, ${ }^{6 \mathrm{f}, \mathrm{g}}$ Amberlyst-15 in ionic liquid, ${ }^{6 \mathrm{~h}} \mathrm{CeCl}_{3} .7 \mathrm{H}_{2} \mathrm{O} / \mathrm{NaI}$ supported on silica gel, ${ }^{6 \mathrm{i}} \mathrm{InBr}_{3},{ }^{6 \mathrm{j}}$ 1-butyl-3methylimidazolium bromide $([\mathrm{bmim}] \mathrm{Br}),{ }^{6 \mathrm{k}} \mathrm{Sc}(\mathrm{OTf})_{3},{ }^{6 \mathrm{l}} \mathrm{CAN}^{6 \mathrm{~m}} \mathrm{ZnCl}_{2}$ under thermal 
conditions, ${ }^{6 \mathrm{n}} \mathrm{AgNO}_{3},{ }^{60}$ sulfated zirconia, ${ }^{6 \mathrm{p}} \mathrm{InCl}_{3}{ }^{6 \mathrm{q}}$ as catalysts. However, many of these methods have some drawbacks such as low yields of the products, ${ }^{6 a, b}$ high temperatures, ${ }^{6 \mathrm{c}}$ long reaction times, $^{6 c}$ and relatively expensive catalysts. ${ }^{6 e, \mathrm{ij}, \mathrm{j}, \mathrm{l}, \mathrm{c}}$ Therefore, the search continues for a better catalyst for the synthesis of 1,5-benzodiazepines in terms of operational simplicity, reusability, economic viability.

The increasing demand for cleaner processes promoted by stringent environment laws requires use of eco-friendly and selective catalysts. Use of inexpensive and non-polluting reagents is highly desirable in recent years and the use of clays as catalyst supports has received considerable attention. ${ }^{7}$ Academic and industrial interest has focused on the use of acid activated montmorillonite K10-supported zinc chloride (zinc montmorillonite, $\mathrm{Zn}^{2+}$-mont); use of this remarkable material has been first reported in $1989 .{ }^{8}$ Examples for $\mathrm{Zn}^{2+}$-mont-mediated organic reactions such as alkylation, preparation of 1,1-diacetates from aldehydes, hydroamination, 3-azaCope rearrangement etc. are well documented in the literature. ${ }^{9}$

In continuation of our interest in developing novel synthetic methodologies, particularly carbon-carbon, carbon-heteratom bond formations, ${ }^{6 \mathrm{~m}, 10}$ and in the use of montmorillonites as environmentally friendly reagents for organic synthesis, we undertook a study of the utility of $\mathrm{Zn}^{2+}$-mont as catalyst for the synthesis of 2,3-dihydro- $1 \mathrm{H}$-1,5-benzodiazepines (Scheme 1). However, to the best of our knowledge, there are no earlier reports on the preparation of 2,3dihydro-1H-1,5-bezodiazepines using $\mathrm{Zn}^{2+}$-mont todate.

\section{Results and Discussion}

At first, we have evaluated the feasibility of the reaction of $o$-phenylenediamine (1a, $1 \mathrm{mmol}$ ) and acetophenone (2a, $2.2 \mathrm{mmol}$ ) using various zinc salts (Table 1) at ambient temperature to afford the corresponding 2,3-dihydro- $1 H$-1,5-benzodiazepine 3a. Some of the examined zinc salts worked as reasonable catalysts, e.g., $\mathrm{ZnBr}_{2}, \mathrm{ZnCl}_{2}, \mathrm{Zn}\left(\mathrm{NO}_{3}\right)_{2}$, etc., while some salts such as zinc hydroxyapatite, $\mathrm{Zn}$ metal (granulated), $\mathrm{Zn}$ dust and $\mathrm{ZnSO}_{4}$ were not effective. Notably, $\mathrm{Zn}^{2+}$-mont (Table 1, entry 8) exhibited superior catalytic activity compared to the unsupported salts such as $\mathrm{ZnBr}_{2}, \mathrm{ZnCl}_{2}$ in terms of regeneration and ease of disposal of the used catalyst. Furthermore, the corresponding homogeneous catalyst (e.g., $\mathrm{ZnCl}_{2}$ ) is least preferred by the industry, because it generates problems such as environmental pollution, handling, safety, corrosion, and tedious work up.

Encouraged by these results, we studied different reaction parameters. The optimum product yield was obtained with a 1:2.2 ratio of $o$-phenylenediamines 1 to ketones 2 . No reaction was observed when $o$-phenylenediamine 1a reacted with acetophenone 2a under similar conditions in the absence of $\mathrm{Zn}^{2+}$-mont as promotor even after stirring for 2 days,. 


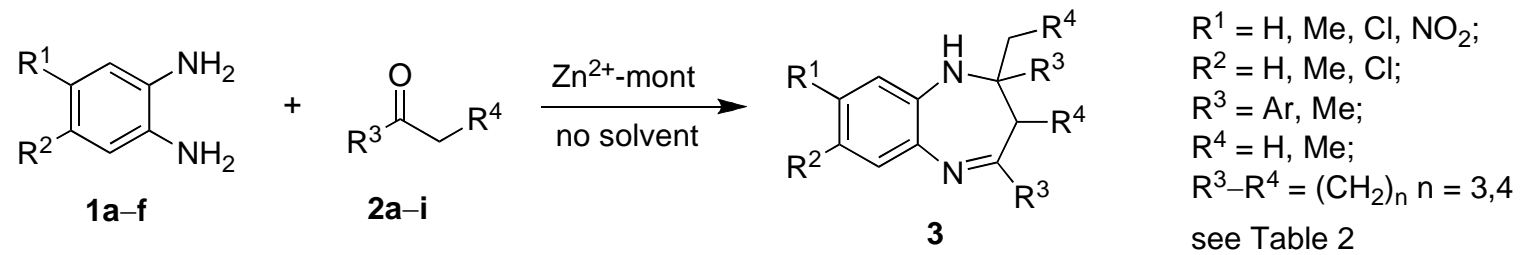

Scheme 1

Table 1. Comparison of $\mathrm{Zn}$ salts as catalysts for the cyclocondensation reaction of $o$-phenylenediamine 1a with acetophenone 2a

\begin{tabular}{|c|c|c|c|}
\hline Entry & Catalyst & $\begin{array}{l}\text { Reaction } \\
\text { time }[\mathrm{h}]\end{array}$ & $\begin{array}{c}\text { Isolated } \\
\text { yield } \\
(\%)\end{array}$ \\
\hline 1 & $\mathrm{Zn}(\mathrm{OAc})_{2} \cdot 2 \mathrm{H}_{2} \mathrm{O}$ & 24 & 35 \\
\hline 2 & $\mathrm{Zn}\left(\mathrm{NO}_{3}\right)_{2}$ & 24 & 55 \\
\hline 3 & Zn(l-proline $)_{2}$ & 36 & 42 \\
\hline 4 & $\mathrm{ZnBr}_{2}$ & 24 & 68 \\
\hline 5 & $\begin{array}{l}\text { Zn- } \\
\text { hydroxyapatite }\end{array}$ & 24 & trace \\
\hline 6 & Zn (granulated) & 48 & 0 \\
\hline 7 & $\mathrm{ZnCl}_{2}$ & 12 & 72 \\
\hline 8 & $\mathrm{Zn}^{2+}$-mont & 12 & 81 \\
\hline 9 & Zn (dust) & 48 & trace \\
\hline 10 & $\mathrm{Zn}(\mathrm{OTf})_{2}$ & 24 & 48 \\
\hline 11 & $\mathrm{ZnSO}_{4}$ & 48 & trace \\
\hline
\end{tabular}

After establishing optimized reaction conditions, we synthesized a variety of biologically relevant 2,3-dihydro- $1 \mathrm{H}$-1,5-benzodiazepines 3 using $\mathrm{Zn}^{2+}$-mont at room temperature (Table 2). In all cases, the reactions were clean and complete within 4-24 h.

Electronically divergent acetophenones 2a-e were employed for the synthesis of 2,3dihydro- $1 H$-1,5-benzodiazepines 3 in moderate to excellent yields (55-90\%). Three new products 3ac, 3ad, and 3ae were characterized by IR, NMR, MS and elemental analyses (see Supporting Information). Benzodiazepines 3 were the only products isolated besides starting materials. The procedure was extended to four aliphatic ketones $2 \mathbf{f}-\mathbf{i}$. The reaction of $o$-phenylenediamines 1a-f with acetone (2f) was performed under similar conditions with good yields of the corresponding 2,3-dihydro- $1 H$-1,5-benzodiazepines 3af-ff (62-80\%). It is noteworthy that in the reaction with the unsymmetrical ketone 2-butanone (2g) ring formation occurred regioselectively forming a single product 3ag (78\%). Cyclopentanone (2h) and cyclohexanone (2i) also reacted well affording moderate yields of the corresponding fused benzodiazepines 3ah and 3ai, respectively. The melting points observed for products 3ah and 
3ai match those reported earlier ${ }^{6 \mathrm{~b}}$ but not those given in a recent paper. ${ }^{6 \mathrm{j}}$ The catalyst could be reused for at least three cycles after activation at $120^{\circ} \mathrm{C}$ for $1 \mathrm{~h}$; there was a slight decrease in activity after the third use in the reaction forming 3aa (78\%). The mechanism of the reaction probably involves an intramolecular imine-enamine cyclization promoted by $\mathrm{Zn}^{2+}$-mont as already reported by Jung and coworkers when using polyphosphoric acid or silica. ${ }^{6 c}$

\section{Conclusions}

A novel and efficient method for the synthesis of 2,3-dihydro- $1 H$-1,5-benzodiazepines 3 is described using the solid acid catalyst $\mathrm{Zn}^{2+}$-mont at ambient temperature. The easy work-up procedure, inexpensive recyclable catalyst and good yields make this method a valid contribution to existing methodologies.

Table 2. $\mathrm{Zn}^{2+}$-mont-Catalyzed synthesis of 2,3-dihydro- $1 H$-1,5-benzodiazepines 3 via cyclocondensation of $o$-phenylenediamines 1 and ketones 2

\begin{tabular}{|c|c|c|c|c|c|c|c|c|c|c|c|}
\hline 1 & $\mathrm{R}^{1}$ & $\mathrm{R}^{2}$ & 2 & $\mathrm{R}^{3}$ & $\mathrm{R}^{4}$ & $\begin{array}{c}\text { Reaction } \\
\text { time }[\mathrm{h}]\end{array}$ & 3 & $\begin{array}{c}\text { Yield }^{\mathrm{a}} \\
(\%)\end{array}$ & $\mathrm{mp}\left[{ }^{\circ} \mathrm{C}\right]$ & $\begin{array}{c}\text { mp lit. } \\
{\left[{ }^{\circ} \mathrm{C}\right]}\end{array}$ & lit. \\
\hline $\mathbf{a}$ & $\mathrm{H}$ & $\mathrm{H}$ & $\mathbf{a}$ & $\mathrm{Ph}$ & $\mathrm{H}$ & 12 & aa & $81^{\mathrm{b}}$ & 152 & 150-152 & $6 \mathrm{l}$ \\
\hline $\mathbf{a}$ & $\mathrm{H}$ & $\mathrm{H}$ & b & $4-\mathrm{MeC}_{6} \mathrm{H}_{4}$ & $\mathrm{H}$ & 10 & ab & 78 & 98-99 & 98-99 & $6 \mathrm{~m}$ \\
\hline $\mathbf{a}$ & $\mathrm{H}$ & $\mathrm{H}$ & c & $4-\mathrm{ClC}_{6} \mathrm{H}_{4}$ & $\mathrm{H}$ & 10 & ac & 90 & $143-145$ & - & - \\
\hline $\mathbf{a}$ & $\mathrm{H}$ & $\mathrm{H}$ & d & $4-\mathrm{BrC}_{6} \mathrm{H}_{4}$ & $\mathrm{H}$ & 16 & ad & 76 & 145-146 & - & - \\
\hline $\mathbf{a}$ & $\mathrm{H}$ & $\mathrm{H}$ & e & 4- $-\mathrm{IC}_{6} \mathrm{H}_{4}$ & $\mathrm{H}$ & 12 & ae & 55 & $143-144$ & - & - \\
\hline b & $\mathrm{Me}$ & $\mathrm{H}$ & $\mathbf{a}$ & $\mathrm{Ph}$ & $\mathrm{H}$ & 24 & ba & 75 & 90 & 92 & $6 \mathrm{k}$ \\
\hline c & $\mathrm{Me}$ & $\mathrm{Me}$ & $\mathbf{a}$ & $\mathrm{Ph}$ & $\mathrm{H}$ & 18 & ca & 68 & 114 & 114-116 & $6 \mathrm{~g}$ \\
\hline c & $\mathrm{Me}$ & $\mathrm{Me}$ & b & 4- $\mathrm{MeC}_{6} \mathrm{H}_{4}$ & $\mathrm{H}$ & 24 & cb & 72 & 102-103 & $102-103$ & $6 \mathrm{~m}$ \\
\hline d & $\mathrm{Cl}$ & $\mathrm{Cl}$ & $\mathbf{a}$ & $\mathrm{Ph}$ & $\mathrm{H}$ & 10 & da & 85 & $158-160$ & $158-160$ & $6 \mathrm{~m}$ \\
\hline d & $\mathrm{Cl}$ & $\mathrm{Cl}$ & b & $4-\mathrm{MeC}_{6} \mathrm{H}_{4}$ & $\mathrm{H}$ & 12 & db & 66 & 179-180 & 179-180 & $6 \mathrm{~m}$ \\
\hline d & $\mathrm{Cl}$ & $\mathrm{Cl}$ & c & $4-\mathrm{ClC}_{6} \mathrm{H}_{4}$ & $\mathrm{H}$ & 12 & dc & 82 & 199-200 & 199-200 & $6 \mathrm{~m}$ \\
\hline $\mathbf{a}$ & $\mathrm{H}$ & $\mathrm{H}$ & f & $\mathrm{Me}$ & $\mathrm{H}$ & 5 & af & 77 & 121 & $120-121$ & $6 \mathrm{~b}$ \\
\hline b & $\mathrm{Me}$ & $\mathrm{H}$ & f & $\mathrm{Me}$ & $\mathrm{H}$ & 7 & bf & 67 & 126 & $127-129$ & $6 \mathrm{j}$ \\
\hline c & $\mathrm{Me}$ & $\mathrm{Me}$ & f & $\mathrm{Me}$ & $\mathrm{H}$ & 8 & cf & 62 & 113 & $112-114$ & $6 \mathrm{j}$ \\
\hline d & $\mathrm{Cl}$ & $\mathrm{Cl}$ & f & $\mathrm{Me}$ & $\mathrm{H}$ & 5 & df & 78 & 92-94 & $92-94$ & $6 \mathrm{~m}$ \\
\hline e & $\mathrm{Cl}$ & $\mathrm{H}$ & f & $\mathrm{Me}$ & $\mathrm{H}$ & 4 & ef & 80 & 90 & $90-92$ & $6 j$ \\
\hline f & $\mathrm{NO}_{2}$ & $\mathrm{H}$ & f & $\mathrm{Me}$ & $\mathrm{H}$ & 10 & ff & 64 & 116 & $113-114$ & $6 j$ \\
\hline $\mathbf{a}$ & $\mathrm{H}$ & $\mathrm{H}$ & g & Et & $\mathrm{H}$ & 8.5 & ag & 78 & 137 & 137-139 & $6 \mathrm{j}$ \\
\hline $\mathbf{a}$ & $\mathrm{H}$ & $\mathrm{H}$ & h & $\left(\mathrm{CH}_{2}\right)_{3}$ & & 12 & ah & 55 & 74 & $72-74$ & $6 \mathrm{~b}$ \\
\hline $\mathbf{a}$ & $\mathrm{H}$ & $\mathrm{H}$ & $\mathbf{i}$ & $\left(\mathrm{CH}_{2}\right)_{4}$ & & 14 & ai & 68 & 98 & 97-99 & $6 \mathrm{~b}$ \\
\hline
\end{tabular}

${ }^{\mathrm{a}}$ Isolated yields after column chromatography. ${ }^{\mathrm{b}}$ Yield after third use of the same catalyst sample: $78 \%$. 


\section{Experimental Section}

$\mathrm{Zn}^{2+}$-mont was prepared according to the literature procedure. ${ }^{9 \mathrm{~d}}$ All reagents were obtained from commercial sources and used without further purification. Solvents for chromatography were distilled before use. ${ }^{1} \mathrm{H}$ and ${ }^{13} \mathrm{C}$ NMR spectra of $\mathrm{CDCl}_{3}$ solutions were recorded on Varian FT$200 \mathrm{MHz}$ (Gemini) and Bruker UXNMR FT-300 MHz (Avance) instruments. Electron-impact (EI) mass spectra were recorded on a VG $7070 \mathrm{H}$ Micromass mass spectrometer at $200^{\circ} \mathrm{C}, 70$ $\mathrm{eV}$. Elemental analyses were performed by Elementar analyzer Vario EL. Melting points were recorded on an Electrothermal melting point apparatus. The IR spectra (using $\mathrm{KBr}$ pellets) were obtained with a Perkin Elmer 240-C instrument. The reactions were monitored by TLC using pre-coated plates (Merck, silica gel 60F-254 on glass). Column chromatography was performed using Acme silica gel (100-200 mesh).

CAUTION: o-phenylenediamines are toxic to work with and hazardous to the environment.

\section{Typical procedure}

A mixture of an $o$-phenylenediamine $1(1.0 \mathrm{mmol})$, a ketone $\mathbf{2}(2.2 \mathrm{mmol})$ and $\mathrm{Zn}^{2+}$-mont (50 $\mathrm{mg}$ ) was vigorously stirred at room temperature for the time specified in Table 2. After completion of the reaction, the reaction mixture was diluted with ethyl acetate $(5 \mathrm{~mL})$, the catalyst was filtered off and washed with ethyl acetate $(2 \times 5 \mathrm{~mL})$. The combined filtrates were concentrated under reduced pressure to afford the crude product 3 , which was further purified by column chromatography (silica gel; ethyl acetate/n-hexane 1:4). All products 3 were recrystallized from ethanol and characterized by comparision of the NMR and mass spectra with those of authentic samples. ${ }^{6}$ See also Supporting Information for 3ac, 3ad, and 3ae.

2,4-Bis(4-chlorophenyl)-2-methyl-2,3-dihydro-1H-1,5-benzodiazepine (3ac). Pale yellow crystals, mp 143-145 ${ }^{\circ} \mathrm{C}$. IR (KBr): $\tilde{v}$ 3269, 1636, 1593, $765 \mathrm{~cm}^{-1} .{ }^{1} \mathrm{H}$ NMR (300 MHz, $\mathrm{CDCl}_{3}$ ): $\delta 1.70$ (s, 3H), 2.79 (d, $J=13.28 \mathrm{~Hz}, 1 \mathrm{H}$ ), 2.97 (d, $J=13.28 \mathrm{~Hz}, 1 \mathrm{H}$ ), 3.25 (br s, NH, 1H), 6.68-6.75 (m, 1H), 6.92-7.02 (m, 1H), 7.12-7.20 (m, 5H), 7.38-7.52 (m, 5H). ${ }^{13} \mathrm{C}$ NMR $\left(75 \mathrm{MHz}, \mathrm{CDCl}_{3}\right): \delta 29.7,42.8,73.4,121.4,121.9,126.6,127.0,128.2,128.3,128.6$, 133.0,137.5, 137.7, 139.8, 145.8, 165.9. ESI MS: $m / z=381$ (100), 350 (9), 102 (41), 85 (5). Anal. Calc. for $\mathrm{C}_{22} \mathrm{H}_{18} \mathrm{Cl}_{2} \mathrm{~N}_{2}$ (381.30): C, 69.30; H, 4.76; N, 7.35; Found: C, 69.29; H, 4.78; N, 7.31.

2,4-Bis(4-bromophenyl)-2-methyl-2,3-dihydro-1H-1,5-benzodiazepine (3ad). Yellow solid, mp 145-146 ${ }^{\circ} \mathrm{C}$. IR (KBr): $\tilde{v}$ 3325, 1640, 1589, 1198, $574 \mathrm{~cm}^{-1} .{ }^{1} \mathrm{H}$ NMR (200 MHz, $\left.\mathrm{CDCl}_{3}\right): \delta$ 1.72 (s, 3H), 2.65 (br s, NH, 1H), 2.87 (d, $J=12.84$ Hz, 1H), 3.00 (d, $J=13.60 \mathrm{~Hz}, 1 \mathrm{H}$ ), 6.976.98 (m, 1H), 7.00-7.04 (m, 6H), 7.18-7.24 (m, 1H), 7.45-7.48 (m, 4H). EI MS: m/z = 470 (5), 458 (5), 350 (7.5), 313 (8.7), 273 (15), 186 (65), 173 (25), 141 (43.7), 108 (100), 80 (50), 53 (18.7). Anal. Calc. for $\mathrm{C}_{22} \mathrm{H}_{18} \mathrm{Br}_{2} \mathrm{~N}_{2}$ (470.20): C, 56.20; H, 3.86; N, 5.96; Found: C, 56.19; H, 3.89; N, 5.92 .

2,4-Bis(4-iodophenyl)-2-methyl-2,3-dihydro-1H-1,5-benzodiazepine (3ae). Pale yellow crystals, mp 143-144 ${ }^{\circ} \mathrm{C}$. IR (KBr): $\tilde{v}$ 3259, 1636, 1579, $462 \mathrm{~cm}^{-1} .{ }^{1} \mathrm{H}$ NMR (300 MHz, 
$\left.\mathrm{CDCl}_{3}\right): \delta 1.71(\mathrm{~s}, 3 \mathrm{H}), 2.85$ (d, $\left.J=13.60 \mathrm{~Hz}, 1 \mathrm{H}\right), 2.99$ (d, $\left.J=12.84 \mathrm{~Hz}, 1 \mathrm{H}\right), 3.32$ (br s, NH), 6.73-6.75 (m, 1H), 6.98-7.03 (m, 2H), 7.21-7.33 (m, 5H), 7.53-7.58 (m, 4H). ${ }^{13} \mathrm{C}$ NMR (75 $\left.\mathrm{MHz}, \mathrm{CDCl}_{3}\right): \delta 30.3,43.4,74.1,93.4,97.4,122.1,122.5,127.3,128.2,129.2,129.3,137.8$, 138.0, 139.4, 140.4, 147.6, 166.8. ESI MS: $m / z=565$ (100), 377 (20), 379 (13), 321 (38), 212 (14), 133 (53), 102 (20), 85 (16), 74 (58). Anal. Calc. for $\mathrm{C}_{22} \mathrm{H}_{18} \mathrm{I}_{2} \mathrm{~N}_{2}$ (564.20): C, 46.83; H, 3.22; N, 4.97; Found: C, 46.79; H, 3.19; N, 5.01.

\section{Supplementary Information Available}

Proofs of evidence for Spectral data for compounds 3ac, 3ad, 3ae.

\section{Acknowledgements}

V. R. thanks DIICT, Dr. J. S. Yadav and Council of Scientific Industrial Research (CSIR, India) for a fellowship.

\section{References}

1. (a) Werner, W.; Baumgart, J.; Burckhardt, G.; Fleck, W. F.; Geller, K.; Gutsche, W.; Hanschmann, H.; Messerschmidt, A.; Roemer, W. Biophys. Chem. 1990, 35, 271. (b) Herpin, T. F.; Van Kirk, K. G.; Salvino, J. M.; Yu, S. T.; Labaudinière, R. F. J. Comb. Chem. 2000, 5, 513. (c) Claremon, D. A.; Liverton, N.; Selnick, H. G.; Smith, G. R. PCT Int. Appl. WO 9640653.

2. (a) Atwal, K. S.; Bergey, J. L.; Hedberg, A.; Moreland, S. J. Med. Chem. 1987, 30, 635. (b) Merluzzi, V.; Hargrave, K. D.; Labadia, M.; Grozinger, K.; Skoog, M.; Wu, J. C.; Shih, C.K.; Eckner, K.; Hattox, S.; Adams, J.; Rosenthal, A. S.; Faanes, R.; Eckner, R. J.; Koup, R. A.; Sullivan, J. L. Science 1990, 250, 1411. (c) Braccio, M. D.; Grossi, G.; Roma, G.; Vargiu, L.; Mura, M.; Marongiu, M. E. Eur. J. Med. Chem. 2001, 36, 935. (d) Tranquillini, M. E.; Cassara, P. G.; Corsi, M.; Curotto, G.; Donati, D.; Finizia, G.; Pentassuglia, G.; Polinelli, S.; Tarzia, G.; Ursini, A.; Van Amsterdam, F. T. M. Arch. Pharm. 1997, 330, 353.

(e) Benedikta, H.; Puodziunaite, D.; Janciene, R.; Kosychova, L. Arkivoc 2000, (iv), 512.

3. (a) Schutz, H. Benzodiazepines, Springer: Heidelberg, 1982. (b) Landquist, J. K. In Comprehensive Heterocyclic Chemistry, Eds. Katritzky A. R.; Rees, C. W. Pergamon: Oxford, Vol. 1, 1984, p166. (c) Randall, L. O.; Kappel, B. Benzodiazepines; Garattini, S.; Mussini, E.; Randall, L. O., Eds.; Raven Press: New York, 1973, p 27.

4. Haris, R. C.; Straley, J. M. U. S. Patent 1,537,757, 1968; Chem. Abstr. 1970, 73, 100054w. 
5. (a) Essaber, M.; Baouid, A.; Hasnaoui, A.; Benharref, A.; Lavergne, J.-P. Synth. Commun. 1998, 28, 4097. (b) El-Sayed, A. M.; Abdel-Ghany, H.; El-Saghier, A. M. M. Synth. Commun. 1999, 29, 3561. (c) Xu, J. X.; Wu, H. T.; Jin, S. Chin. J. Chem. 1999, 17, 84. (d) Zhang, X. Y.; Xu, J. X.; Jin, S. Chin. J. Chem. 1999, 17, 404. (e) Reddy, K. V. V.; Rao, P. S.; Ashok, D. Synth. Commun. 2000, 30, 1825.

6. (a) Herbert, J. A. L.; Suschitzky, H. J. Chem. Soc., Perkin Trans. 1 1974, 2657. (b) Morales, H. R.; Ulbarela, B. A.; Contreras, R. Heterocycles 1986, 24, 135. (c) Jung, D. I.; Choi, T. W.; Kim, Y. Y.; Kim, I. S.; Park, Y. M.; Lee, Y. G.; Jung, D. H. Synth. Commun. 1999, 29, 1941. (d) Balakrishna, M. S.; Kaboudin, B. Tetrahedron Lett. 2001, 42, 1127. (e) Curini, M.; Epifano, F.; Marcotullio, M. C.; Rosati, O. Tetrahedron Lett. 2001, 42, 3193. (f) Kaboudin, B.; Navaee, K. Heterocycles 2001, 55, 1443. (g) Pozarentzi, M.; Stephanatou, J. S.; Tsoleridis, C. A. Tetrahedron Lett. 2002, 43, 1755. (h) Yadav, J. S.; Reddy, B. V. S.; Eshwaraiah, B.; Anuradha, K. Green Chem. 2002, 4, 592. (i) Sabitha, G.; Reddy, G. S. K. K.; Reddy, K. B.; Reddy, N. M.; Yadav, J. S. Adv. Synth. Catal. 2004, 346, 921. (j) Yadav, J. S.; Reddy, B. V. S.; Kumar, S. P.; Nagaiah, K. Synthesis 2005, 480. (k) Jarikote, D. V.; Siddiqui, S. A.; Rajagopal, R.; Daniel, T.; Lahoti, R. J.; Srinivasan, K. V. Tetrahedron Lett. 2003, 44, 1835. (l) De, S. K.; Gibbs, R. A. Tetrahedron Lett. 2005, 46, 1811. (m) Varala, R.; Ramu, E.; Sreelatha, N.; Adapa, S. R. Synlett 2006, 1009. (n) Pasha, M. A.; Jayashankara, V. P. Heterocycles 2006, 68, 1017. (o) Kumar, R.; Chaudhary, P.; Nimesh, S.; Verma, A. K.; Chandra, R. Green Chem. 2006, 8, 519. (p) Reddy, B. M.; Sreekanth, P. M.; Lakshmanan, P. J. Mol. Catal. A: Chem. 2005, 237, 93. (q) Yadav, J. S.; Reddy, B.V.S.; Satheesh, G.; Srinivasulu, G.; Kunwar, A. C. Arkivoc 2005, (iii), 221.

7. Laszlo, P. Acc. Chem. Res. 1986, 19, 121.

8. Clark, J. H.; Kybett, A. P.; Macquarrie, D. J.; Barlow, S. J.; Landon, P. J. Chem. Soc., Chem. Commun. 1989, 1353.

9. (a) Rhodes, C. N.; Franks, M.; Parkes, G. M. B.; Brown, D. R. J. Chem. Soc. Chem. Commun. 1991, 804. (b) Rhodes, C. N.; Brown, D. R. J. Chem. Soc., Faraday Trans. 1992, 15, 2269. (c) Nagy, N. M.; Jakab, M. A.; Konya, J.; Antus, S. Applied Clay Science. 2002, 21, 213. (d) Shanbhag, G. V.; Halligudi, S. B. J. Mol. Catal. A. Chemical. 2004, 222, 223. (e) Yadav, J. S.; Reddy, B. V. S.; Rasheed, M. A.; Kumar, H. M. S. Synlett 2000, 487.

10. (a) Varala, R.; Ramu, E.; Adapa, S. R. Bull. Chem. Soc. Jpn. 2006, 79, 140, and references cited therein. (b) Varala, R.; Ramu, E.; Sreelatha, N.; Adapa, S. R. Tetrahedron Lett. 2006, 47, 877. (c) Alam, M. M.; Varala, R.; Ramu, E.; Adapa, S. R. Lett. Org. Chem. 2006, 3, 187.

(d) Varala, R.; Ramu, E.; Vijay, K. P.; Adapa, S. R. Chin. J. Chem. 2006, 24, 807. (e) Varala, R.; Sreelatha, N.; Adapa, S. R. Adapa, Synlett 2006, 1549. (f) Varala, R.; Ramu, E.; Adapa, S. R. Synthesis 2006 (in press). (g) Varala, R.; Sreelatha, N.; Adapa, S. R. Bull. Korean Chem. Soc. 2006, 27, 1079. 